Review Article

\title{
Mantel test in population genetics
}

José Alexandre F. Diniz-Filho ${ }^{1}$, Thannya N. Soares ${ }^{2}$, Jacqueline S. Lima ${ }^{3}$, Ricardo Dobrovolski ${ }^{4}$, Victor Lemes Landeiro ${ }^{5}$, Mariana Pires de Campos Telles ${ }^{2}$, Thiago F. Rangel ${ }^{1}$ and Luis Mauricio Bini ${ }^{1}$

${ }^{1}$ Departamento de Ecologia, Universidade Federal de Goiás, Goiânia, GO, Brazil.

${ }^{2}$ Departamento de Biologia Geral, Universidade Federal de Goiás, Goiânia, GO, Brazil.

${ }^{3}$ Programa de Pós-Graduação em Ecologia e Evolução, Universidade Federal de Goiás, Goiânia, GO, Brazil.

${ }^{4}$ Departamento de Zoologia, Universidade Federal da Bahia, Salvador, BA, Brazil.

${ }^{5}$ Departamento de Botânica e Ecologia, Universidade Federal de Mato Grosso, Cuiabá, MT, Brazil.

\begin{abstract}
The comparison of genetic divergence or genetic distances, estimated by pairwise $\mathrm{F}_{\mathrm{ST}}$ and related statistics, with geographical distances by Mantel test is one of the most popular approaches to evaluate spatial processes driving population structure. There have been, however, recent criticisms and discussions on the statistical performance of the Mantel test. Simultaneously, alternative frameworks for data analyses are being proposed. Here, we review the Mantel test and its variations, including Mantel correlograms and partial correlations and regressions. For illustrative purposes, we studied spatial genetic divergence among 25 populations of Dipteryx alata ("Baru"), a tree species endemic to the Cerrado, the Brazilian savannas, based on 8 microsatellite loci. We also applied alternative methods to analyze spatial patterns in this dataset, especially a multivariate generalization of Spatial Eigenfunction Analysis based on redundancy analysis. The different approaches resulted in similar estimates of the magnitude of spatial structure in the genetic data. Furthermore, the results were expected based on previous knowledge of the ecological and evolutionary processes underlying genetic variation in this species. Our review shows that a careful application and interpretation of Mantel tests, especially Mantel correlograms, can overcome some potential statistical problems and provide a simple and useful tool for multivariate analysis of spatial patterns of genetic divergence.
\end{abstract}

Keywords: "Baru" tree, genetic distances, geographical genetics, partial correlation, partial regression.

Received: June 12, 2013; Accepted: October 10, 2013.

\section{Introduction}

The estimation of genetic divergence between individuals from different localities ("populations" hereafter) has been an important component of empirical studies in population genetics. These studies are supported by a strong theoretical basis since the classical papers by S. Wright, R.A. Fisher and G. Malecot, among others (Epperson, 2003). The most popular approaches for estimating divergence include calculation of genetic distances and variance partitioning among and within populations using Wright's $F_{\mathrm{ST}}$ and other related statistics, such as $\mathrm{G}_{\mathrm{ST}}, \mathrm{A}_{\mathrm{ST}}$, $\mathrm{R}_{\mathrm{ST}}, \theta_{\mathrm{ST}}$ and $\phi_{\mathrm{ST}}$ (see Holsinger and Weir, 2009 for a recent review). For instance, the $\mathrm{F}_{\mathrm{ST}}$ gives an estimate of the balance of genetic variability among and within populations, and is an unbiased estimator of divergence between pairs of populations under an island-model in which all populations diverged at the same time and are linked by approximately

Send correspondence to José Alexandre Felizola Diniz-Filho. Universidade Federal de Goiás, Departamento de Ecologia, Caixa Postal 131, Goiânia, GO, Brazil. E-mail: jafdinizfilho@gmail.com. similar migration rates. However, migration rates usually vary proportionally with geographical distances, so that pairwise $\mathrm{F}_{\mathrm{ST}}$ estimates between pairs of populations vary.

Regardless of how genetic divergence among populations is computed, a recurrent goal in landscape genetics is to evaluate the amount of spatial structure in the genetic distance matrix. For instance, it is common to use cluster (such as UPGMA or Neighbor-Joining) and ordination techniques (e.g., Principal Coordinates Analysis) to visualize the relationships among populations based on these matrices (see Lessa, 1990; Felsenstein, 2004). More recent techniques, such as Bayesian approaches (see Balkenhol et al., 2009; Guillot et al., 2009), do not start from pairwise distances, but follow a similar reasoning of establishing clusters based on genetic differentiation among individuals. However, these approaches do not explicitly evaluate the effect of geographic space. By far, the Mantel test is the most commonly used method to evaluate the relationship between geographic distance and genetic divergence (Mantel, 1967; see Manly, 1985, 1997). 
The Mantel test was proposed in 1967 to test the association between two matrices and was first applied in population genetics by Sokal (1979). Despite recent controversies and criticisms about its statistical performance (e.g. Harmon and Glor 2010; Legendre and Fortin, 2010; Guillot and Rousset, 2013) and the existence of more sophisticated and complex approaches to analyze spatial multivariate data, the Mantel test is still widely used. We believe that at least part of the problems associated with this test is due to lack of understanding of basic aspects of the test and misinterpretations in empirical applications.

Here we review the Mantel test and its extensions (Mantel correlogram, partial correlation and regression), discussing how it can be associated with theoretical models in population genetics (i.e., isolation-by-distance and landscape models). Routines of different forms of the Mantel test are widely available in several computer programs for population genetic analyses (Table 1) and in several packages for the R platform (R Development Core Team, 2012). All Mantel tests performed here were conducted using the $\mathrm{R}$ packages vegan (Oksanen et al., 2012) and ecodist (Goslee and Urban, 2007) and a complete script is available from the authors upon request.

We illustrate several applications of the Mantel test using an example based on population genetic divergence among Dipteryx alata populations, the "Baru", an endemic tree widely distributed in the Brazilian Cerrado biome (see Diniz-Filho et al., 2012a,b; Soares et al., 2012). Previous analyses suggested that spatial patterns of genetic variability in this species are due to a combination of isolation-by-distance and range expansion after the last maximum glacial, creating clines in some loci.

\section{Original Formulation}

The Mantel test, as originally formulated in 1967 , is given by

$$
Z_{m}=\sum_{i=1}^{n} \sum_{j=1}^{n} g_{i j} \times d_{i j}
$$

where $g_{i j}$ and $d_{i j}$ are, respectively, the genetic and geographic distances between populations $i$ and $j$, considering $n$ populations. Because $Z_{m}$ is given by the sum of products of distances its value depends on how many populations are studied, as well as the magnitude of their distances. The $Z_{m}$-value can be compared with a null distribution, and Mantel originally proposed to test it by the standard normal deviate (SND), given by

$$
\mathrm{SND}=Z_{m} / \operatorname{var}\left(Z_{m}\right)^{1 / 2}
$$

Table 1 - Some of the softwares available for different approaches based on Mantel tests, including simple Mantel test (S), partial Mantel tests (P) and

\begin{tabular}{|c|c|c|}
\hline Software & Mantel approach & Website \\
\hline Alleles in Space (AIS) & $\mathrm{S}$ & http://www.marksgeneticsoftware.net/AISInfo.htm \\
\hline Arlequin & $\mathrm{S}, \mathrm{P}$ & http://cmpg.unibe.ch/software/arlequin35/Ar135Downloads.html \\
\hline Fstat & $\mathrm{S}, \mathrm{P}$ & http://www2.unil.ch/popgen/softwares/fstat.htm \\
\hline GenAlEx & $\mathrm{S}, \mathrm{P}$ & http://biology.anu.edu.au/GenAlEx/Download.html \\
\hline Genepop & $\mathrm{S}$ & http://genepop.curtin.edu.au/ \\
\hline Genetix & $\mathrm{S}$ & http://kimura.univ-montp2.fr/genetix/ \\
\hline IBD (Isolation by Distance) & $\mathrm{S}, \mathrm{P}$ & http://www.bio.sdsu.edu/pub/andy/IBD.html \\
\hline IBDWS (On line) & $\mathrm{S}, \mathrm{P}$ & $\begin{array}{l}\text { http://www.webcitation.org/query.php?url=http://phage.sdsu.edu/ jensen/\& } \\
\text { refdoi }=10.1186 / 1471-2156-6-13\end{array}$ \\
\hline Mantel Nonparametric Test Calculator & $\mathrm{S}$ & http://www.terc.csiro.au/mantel.htm \\
\hline Mantel-Struct & $\mathrm{S}$ & http://www.marksgeneticsoftware.net/mantel.htm \\
\hline NTSys & $\mathrm{S}, \mathrm{P}$ & http://www.exetersoftware.com/cat/ntsyspc/ntsyspc.html \\
\hline PASSaGE & $\mathrm{S}, \mathrm{P}, \mathrm{C}$ & http://www.passagesoftware.net/download.php \\
\hline PC-Ord & $\mathrm{S}, \mathrm{P}$ & http://people.oregonstate.edu/ mccuneb/pcord.htm \\
\hline Quiime & $\mathrm{S}, \mathrm{P}, \mathrm{C}$ & http://qiime.org/index.html \\
\hline $\mathrm{R}$ - package vegan & $\mathrm{S}, \mathrm{P}, \mathrm{C}$ & http://vegan.r-forge.r-project.org/ \\
\hline $\mathrm{R}$ - package ade 4 & S & http://pbil.univ-lyon1.fr/ADE-4/ \\
\hline $\mathrm{R}$ - package ecodist & $\mathrm{S}, \mathrm{P}, \mathrm{C}$ & http://cran.r-project.org/web/packages/ecodist/index.html \\
\hline SAM & $\mathrm{S}, \mathrm{C}$ & http://www.ecoevol.ufg.br/sam/ \\
\hline Spagedi & $\mathrm{S}$ & http://ebe.ulb.ac.be/ebe/SPAGeDi.html \\
\hline TFPGA & $\mathrm{S}$ & http://www.marksgeneticsoftware.net/tfpga.htm \\
\hline ZT & $\mathrm{S}, \mathrm{P}$ & http://bioinformatics.psb.ugent.be/webtools/zt/ \\
\hline
\end{tabular}
correlograms (C), and the website where they can be found. 
where $\operatorname{var}\left(Z_{m}\right)$ is the variance of the $Z_{m}$ (see Mantel, 1967 and Manly, 1985 for detailed formulas). Later, however, Mielke (1978) showed that this formulation is biased, working well only for large sample sizes, and suggested that a null distribution must be obtained empirically by permuting rows and columns of one of the distance matrices.

Thus, the idea underlying Mantel's randomization test is that if there is a relationship between matrices $\mathbf{G}$ and D, the sum of products $Z_{m}$ will be relatively high, and randomizing rows and columns will destroy this relationship so that $Z_{m}$ values, after randomizations, will tend to be lower than the observed. If one generates, say, 999 values and none of the randomized $Z_{m}$-values is higher than the observed, it is possible to conclude that the chance to observe a $Z_{m}$-value as high as the observed by chance alone is $1 / 999+1$ (the 1 is the observed, which is conservatively added to both the numerator and denominator). This is then the p-value from Mantel test.

One can also use a standardized version of the Mantel's test $\left(Z_{N}\right)$ :

$$
Z_{N}=\frac{\sum_{i=1}^{n} \sum_{j=1}^{n}\left(g_{i j}-\overline{\mathbf{G}}\right) \times\left(d_{i j} \times \overline{\mathbf{D}}\right)}{\operatorname{var}(\mathbf{G})^{1 / 2} \times \operatorname{var}(\mathbf{D})^{1 / 2}}
$$

using the means $(\overline{\mathbf{G}}$ and $\overline{\mathbf{D}})$ and the variances $(\operatorname{var}(\mathbf{G})$ and $\operatorname{var}(\mathbf{D}))$ of the matrices $\mathbf{G}$ and $\mathbf{D}$. The standardized version of Mantel's test $\left(Z_{N}\right)$ is actually the Pearson correlation $r$ between the standardized elements of the matrices $\mathbf{G}$ and $\mathbf{D}$. $Z_{N}$ values close to 1 indicate that an increase in geographic distance between populations $i$ and $j$ is related with an increase in genetic distances between these populations. $Z_{N}$ values close to -1 indicate de opposite pattern, and $Z_{N}$ values close to zero indicate that there is no relationship between the two matrices. Notice also that if the two matrices $\mathbf{G}$ and $\mathbf{D}$ are standardized prior to the analysis (so that the mean is equal to 0 and variance is equal to 1) Mantel original $Z_{m}$ and standardized $Z_{N}$ have exactly the same value. For simplicity of notation, this standardized Mantel test $Z_{N}$ will be referred to hereafter as Mantel correlation $r_{m}$.

The dataset for Dipteryx alata populations used throughout the text consists of genotypes based on 8 microsatellite loci of 644 individuals collected in 25 populations of the Brazilian Cerrado (States of Goiás, Mato Grosso, Mato Grosso do Sul, Minas Gerais and Tocantins, Figure 1; see Diniz-Filho et al., 2012a,b for details). The overall $\mathrm{F}_{\mathrm{ST}}$ was equal to 0.254 , indicating a spatial heterogeneity among populations. We built matrices of genetic distances among population by calculating pairwise $\mathrm{F}_{\mathrm{ST}}$ estimated by an Analysis of Variance of Allele Frequencies (Holsinger and Weir, 2009) and Nei's genetic distances (these two genetic distances are strongly correlated: $r_{m}=0.868$; $\mathrm{p}<0.001)$. We then correlated these genetic distance matrices with pairwise geographic distances (measured in kilometers) between populations. Results of Mantel tests are

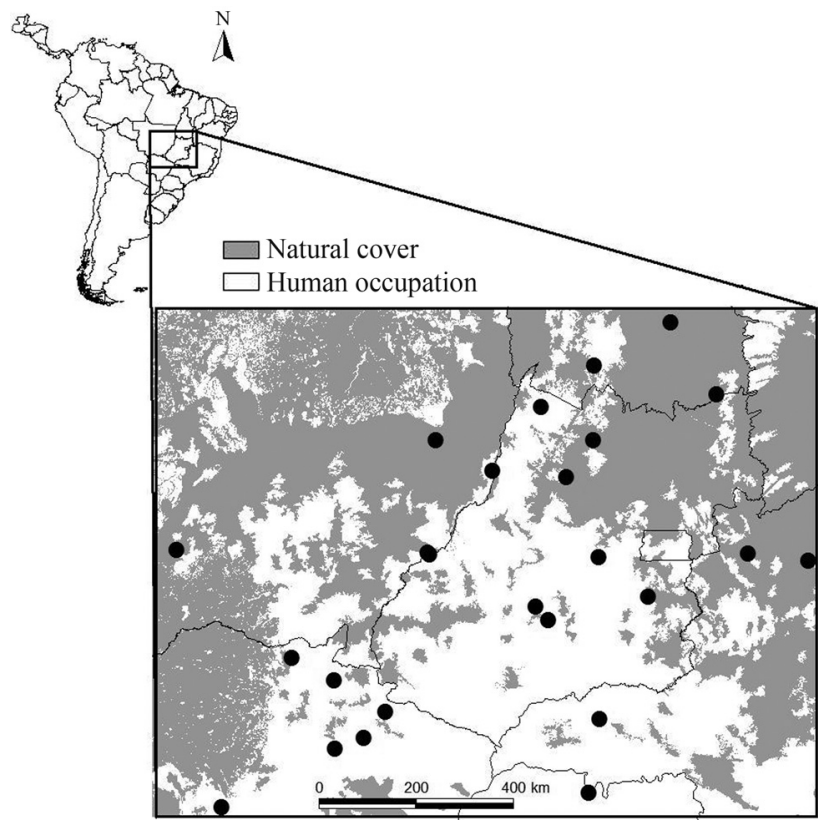

Figure 1 - The twenty-five populations of Dipterx alata, the "Baru" tree, for which 644 individuals were genotyped for 8 microsatellite loci, used in the examples for the Mantel test. Dark regions represent remnants of natural vegetation.

qualitatively the same using pairwise $\mathrm{F}_{\mathrm{ST}}$ or Nei's genetic distances, so a $\mathbf{G}$ matrix is hereafter given by the pairwise $\mathrm{F}_{\mathrm{ST}}$.

The first and simplest application of the Mantel test is to correlate genetic (G) and geographic (D) distances, seeking for spatial pattern of genetic variation. The Mantel correlation between $\mathbf{G}$ and $\mathbf{D}$ matrices was equal to 0.499 . The scatterplot between elements in $\mathbf{G}$ and $\mathbf{D}$ matrices showed a linear relationship between genetic and geographic distances (Figure 2). Performing 4999 randomizations of the rows and columns of $\mathbf{G}$ generated the distribution of correlations under the null hypothesis. Out of these 4999 values, none was larger than the observed value of 0.499 , so that the chance of obtaining a value as large as the observed is smaller than $1 / 5000$, indicating a $p$-value of 0.0002 . Thus, we conclude that nearby populations tend to be genetically more similar than expected by chance, and genetic differences increase linearly with geographic distances.

\section{Two Useful Extensions: Mantel Correlograms And Partial Mantel Tests}

\section{Mantel correlograms}

The Mantel correlation, as shown in Figure 2, shows the overall relationship between matrices $\mathbf{G}$ and $\mathbf{D}$. However, it is often interesting to study the relationship between genetic and geographic distances across space, especially if this relationship is not linear. Thus, the matrix $\mathbf{D}$ can be divided into several sub-matrices, each one describing pairs of populations within a bounded interval of geographic dis- 


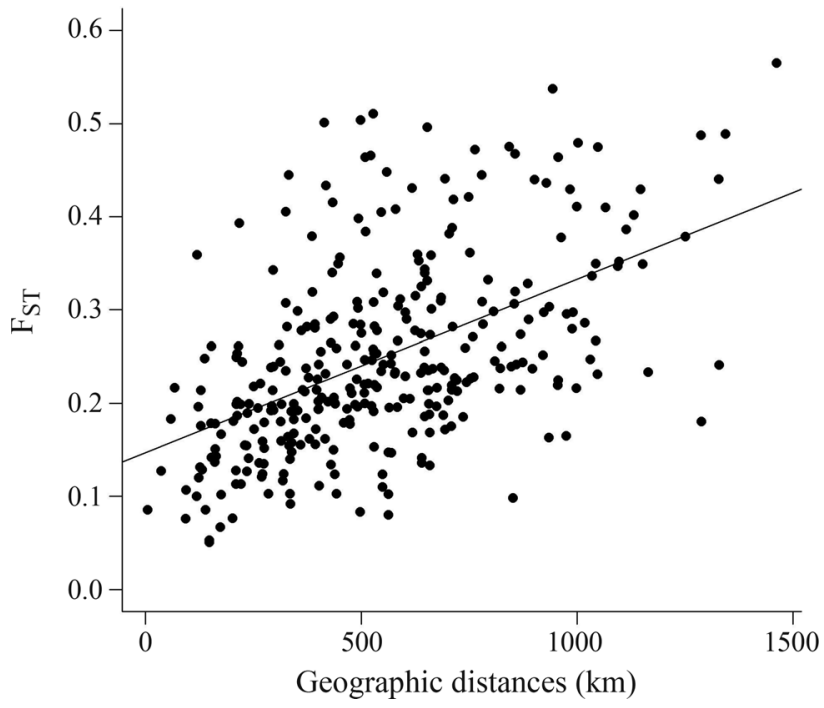

Figure 2 - Relationship between pairwise $\mathrm{F}_{\mathrm{ST}}$ and geographic distances $(r=0.499)$ for the 25 "Baru" populations.

tances. Specifically, this is done to describe possible variations in the correlation between genetic and geographic distances. These matrices, called here $\mathbf{W}_{k}$, express in a binary form $(0 / 1$ values $)$ if pairs of populations are connected (a value of 1), or not (a value of 0 ), within a given geographic distance range, usually referred as "distance class" $k$. To analyze the variation of correlation coefficients across space it is, however, necessary to create multiple nonoverlapping and contiguous distance classes. Thus, several Mantel correlations are obtained by performing a Mantel test between $\mathbf{G}$ and the matrices $\mathbf{W}_{1}, \mathbf{W}_{2}, \mathbf{W}_{3}, \ldots, \mathbf{W}_{k}$. Finally, the Mantel correlogram is constructed by plotting Mantel correlations between $\mathbf{G}$ and each $\mathbf{W}$ against the mid-point of the respective distance class $k$ (Oden and Sokal, 1986; Legendre and Legendre, 2012). The definition of distance classes, both in terms of the total number of classes and their upper and lower limits, is somewhat arbitrary and depends on the spatial distribution of the populations. A "rule of thumb" suggests about four to five classes for 20 populations.

From a statistical point of view it is recommendable to keep the number of links (pairs of populations) within each matrix $\mathbf{W}$ approximately constant, which may require unequal distance intervals (e.g., 0-100 km, 100-250 km, 250-500 km, 500-2000 km, see Sokal and Oden, 1978a,b for a discussion). The most important issue about correlograms is that they should capture a continuous distribution in geographic space. Thus, it is desirable to have a large number of classes. However, one must keep in mind that, if the number of populations is relatively small, or if the populations are distributed irregularly across space (e.g. aggregated in clusters), it may not be possible to use a large number of distance classes. This is so because there may not be enough pairs of populations within a given distance class to provide a reliable estimate of the correlation.
For the "Baru" populations, a correlogram with five geographic distances classes indicated that populations distant by $156 \mathrm{~km}$ (first distance class: from $0 \mathrm{~km}$ to $318 \mathrm{~km}$ ) tend to be similar $\left(r_{m}=0.337 ; \mathrm{p}<0.001\right.$ with 4999 permutations) (Figure 3a). The Mantel correlation decreased more or less linearly up to a value of $-0.333(p<0.001)$ in the last distance class, when populations were approximately $1120 \mathrm{~km}$ apart. As discussed earlier, negative correlation values indicate that populations that are located at a given distance apart tend to be genetically dissimilar. Notice, however, that the Mantel correlations in both the first and last distance classes were not very high (i.e., - 0.33 ), indicating that the spatial structure is not strong (remember that the overall Mantel test is 0.499 , so that only about $24.9 \%$ (i.e. $0.499^{2}$ ) of the genetic divergence is explained by geographic distance - see below).

It is also possible to compute the mean $\mathrm{F}_{\mathrm{ST}}$ within each distance class and plot it against the mean value of the class (Figure $3 b$ ). This is sometimes called distogram and provides an interesting and more direct visual evaluation of spatial patterns in genetic structure. For the "Baru" dataset, when nearby populations in the first distance class were compared, the mean $\mathrm{F}_{\mathrm{ST}}$ was 0.224 (smaller than the overall value of 0.367 ), whereas in the last distance class the mean $\mathrm{F}_{\mathrm{ST}}$ was equal to 0.522 , which is higher than the mean value.

Thus, the correlogram and the distogram showed a continuous and linear decrease of genetic similarity (a
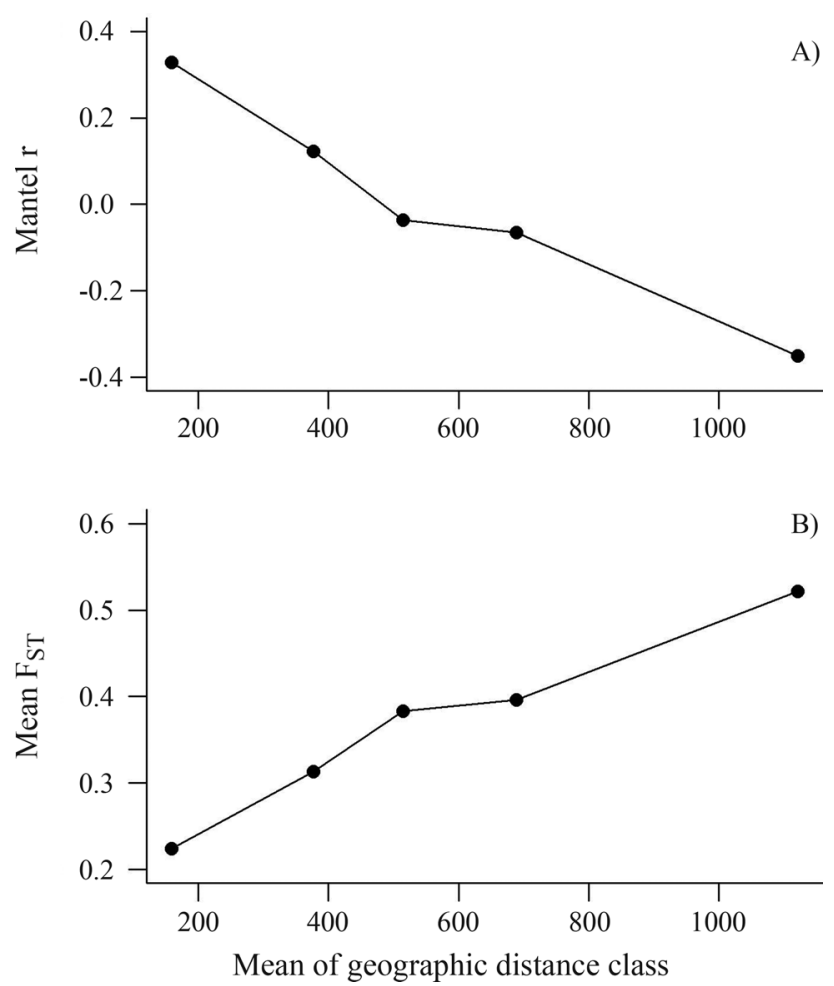

Figure 3 - Mantel correlogram (A) and distogram (B), the latter one given by the mean $\mathrm{F}_{\mathrm{ST}}$ in each distance class. 
higher mean $\mathrm{F}_{\mathrm{ST}}$, and a lower Mantel correlation) when geographic distance increased (Figure 2a). This result is expected when there is a clinal pattern of genetic variation in the studied region (i.e., when allele frequencies decrease or increase in a directional way). Spatial clines can arise by selection along environmental gradients (unlike in the case of microsatellite markers), and/or by range expansions or diffusion of genes through space in migratory events or allelic surfing. Indeed, previous analyses suggest that patterns of genetic variation in "Baru" are related to range expansions from north to south, tracking climate changes after the last glacial maximum (see Diniz-Filho et al., 2012b).

Other more complex patterns can be detected using correlograms, and perhaps the most common pattern observed in nature is an exponential-like decrease in which there are high Mantel correlations in the first distance classes, which tend to decrease and stabilize after a given distance class, indicating that there are patches of genetic variation or similarity. These patches can be caused by several factors, including different environments driving genetic variation (again unlike in the case of microsatellites), or the subdivision of the studied region by barriers, or simple isolation-by-distance (see below). The geographic distance at which the Mantel correlation is zero or nonsignificant indicates the size of the patch, and this can be useful for understanding population and genetic dynamics in space (see Sokal and Wartenberg, 1983; Sokal et al., 1997). Patch size can also be used for establishing more efficient approaches in conservation genetics, allowing to estimate regions within which genetic variability is similar (see Diniz-Filho and Telles, 2002, 2006).

When exponential-like correlograms appear, the overall Mantel test may be a poor estimate of the spatial pattern because it assumes a linear correlation between matrices. Thus it is important to check for non-linearity and heteroscedastic relationships between geographic and genetic distances with a simple scatterplot before interpreting the result of a global Mantel test. An even safer alternative would consist in using correlograms instead of the simple Mantel test (see Borcard and Legendre, 2012).

Finally, it is also important to highlight that, despite recent discussions on the validity of the Mantel test (especially of the partial Mantel tests, see below), the Mantel correlogram deserves its place in the ecologist's "toolbox". For instance, Borcard and Legendre (2012) recently used several simulations to show that the statistical performance of a Mantel correlogram, for both Type I and Type II error rates, is reliable.

\section{Partial Mantel tests}

Another possibility for using the Mantel test is to compare the relationship between two matrices, but taking into account the effect of a third one (usually the geographical distances), as originally proposed by Smouse et al. (1986). When analyzing spatially distributed data, the main issue is to find out if the two matrices are "causally" related (i.e., in the sense that they indicate an ecological or evolutionary process), or if the observed relationship appears only because both variables are spatially structured by intrinsic effects (i.e., distance-structured dispersal causing more similarity between neighboring populations).

When one is interested in evaluating the statistical correlation between two variables (say, an allele frequency and temperature) whose values are spatially distributed, the most common (and statistically sound) approach is to apply spatial regression ,methods (see Diniz-Filho et al., 2009 for a review using genetic data and Perez et al., 2010 for an application). However, when the hypotheses are specified in terms of distance matrices, such as in the case of isolation-by-distance and many landscape models (see Wagner and Fortin, 2013), the most popular approach is to apply partial Mantel tests (see Legendre and Legendre, 2012 for a review).

There are several forms of partial Mantel tests (see Smouse et al., 1986; Oden and Sokal, 1992; Legendre and Legendre, 2012), but the general reasoning is to evaluate how two matrices are correlated after controlling, or keeping statistically constant, the effect of other matrices (see Sokal et al., 1986, 1989, for initial applications). In a first approach, it is possible to calculate the partial correlation between matrices $\mathbf{G}$ and $\mathbf{E}$ (where $\mathbf{E}$ is a distance matrix that one wants to correlate with $\mathbf{G}$, keeping matrix $\mathbf{D}$ constant). The partial correlation is given by

$\left.\left.r_{(\mathrm{GE|D})}=r_{m(\mathrm{GE})}-r_{m(\mathrm{ED})} r_{m(\mathrm{GD})} /\left[\left(1-r_{m(\mathrm{ED})}\right)^{2}\right)^{1 / 2}\right]\left[\left(1-r_{m(\mathrm{GD})}\right)^{2}\right)^{1 / 2}\right]$

where $Z_{N(\mathrm{GE})}$ is, for instance, the correlation coefficient between matrices $\mathbf{G}$ and $\mathbf{E}$ and $r_{(\mathrm{GE} \mid \mathrm{D})}$ is the correlation between $\mathbf{G}$ and $\mathbf{E}$, after taking $\mathbf{D}$ into account.

To illustrate these approaches with the "Baru" dataset, it is necessary to generate other explanatory matrices. First, for each locality, we obtained the altitude and 19 bioclimatic variables from WorldClim (Hijmans et al., 2005 ) and, after standardizing each variable to zero mean and unity variance, an environmental (Euclidean) distance matrix for all possible pairwise combinations of the local populations was obtained. This matrix (E) expresses then the environmental (mainly climatic) differences between populations. Second, we also estimated the amount of natural habitats remaining between pairs of populations, as the proportion of natural habitats in a $10 \mathrm{~km}$ wide "corridor" linking two populations (a matrix R). This matrix was derived from land use data obtained using the vegetation cover maps of the Brazilian biomes at the 1:250.000 spatial scale, based on compositions of the bands 3,4 and 5 of Landsat 7 ETM+ images of the year 2002 (see Diniz-Filho et al., 2012a).

A simple Mantel correlation revealed that $\mathrm{F}_{\mathrm{ST}}$ is not correlated to the proportion of the natural remnants matrix $\mathbf{R}\left(r_{m}=-0.23 ; \mathrm{p}=0.142\right)$, and that this matrix is not spatially correlated $\left(r_{m}=-0.075 ; \mathrm{p}=0.552\right)$. Thus, no further partial 
analyses were needed (Dutilleul, 1993). However, $\mathrm{F}_{\mathrm{ST}}$ is significantly correlated with environmental distances $\mathbf{E}$ according to a simple Mantel test $\left(r_{m}=0.302 ; \mathrm{p}=0.008\right)$. However, we already know that genetic divergence is spatially patterned $\left(r_{m}=0.499\right)$ and there is also a very strong spatial pattern in environmental variation $\mathbf{E}\left(r_{m}=0.838\right.$; $\mathrm{p}<0.001$ ). Thus, the main issue is to test if there is a correlation between $\mathbf{G}$ and $\mathbf{E}$, after taking the geographic distances (matrix D) into account. This relationship is not expected for neutral markers as microsatellites, except if one considers that these loci are linked with adaptive ones.

Indeed, the partial correlation between $\mathbf{G}$ and $\mathbf{E}$, after taking into account geographic distances $\mathbf{D}$, was equal to$r_{m \text { (GE|D) }}=-0.248(\mathrm{p}=0.956)$, so the relationship between genetic and environment disappeared when geographic structure common to both matrices was accounted for (as in principle expected for neutral markers, as pointed out above). First, it is possible to quantify the relationships between $\mathrm{F}_{\mathrm{ST}}$ and geographic distance $\mathbf{D}$ and environmental distance $\mathbf{E}$ by partial coefficients of determination, disentangling the amount of variation explained by each predictor matrix and their shared contribution (see Pellegrino et al., 2005 for an application in a phylogeographical context). In the "Baru" example, the geographic distances explained $24.9 \%$ of the variation in $\mathrm{F}_{\mathrm{ST}}$ (the square of the Mantel correlation, $r_{m}{ }^{2}$ equal to 0.499 ), whereas the effect of environment was equal to $9.09 \%$. Using a standard multiple regression framework, if the matrices $\mathbf{E}$ and $\mathbf{D}$ are used as explanatory matrices to explain $\mathrm{F}_{\mathrm{ST}}$, the overall $R_{m}{ }^{2}$ is equal to 0.295 . The sum of the $r_{m}{ }^{2}$ is slightly larger than the overall $R_{m}{ }^{2}$ and, therefore, there is a small shared fraction (4.4\%). The unique effects of geographic and environmental distances are equal to 0.204 and 0.046 , respectively. This result reveals that about half of the small explanatory power of environmental distances was due to spatial patterns (in agreement with the results of the partial correlation shown above).

Finally, it is also possible to generalize the multiple regression approach and evaluate simultaneously the effects of several explanatory matrices, a framework called Multiple Regression on Distance Matrices (MRM; Lichstein, 2007). Using the "Baru" dataset, we can evaluate the "effects" of the explanatory distance matrices (D, E and R) on the genetic divergence estimated by $\mathrm{F}_{\mathrm{ST}}$. In this case, these matrices explained $32.1 \%$ of the variation in genetic divergence, and only the standardized partial regression coefficient of geographic distances was significant at $\mathrm{p}<0.001$ ( $\mathrm{p}$-value for $\mathbf{E}$ was equal to 0.111 and for $\mathbf{R}$ equal to 0.239 ). The results are thus similar to all previous Mantel tests that did not show partial effects of the environment or proportion of natural remnants on genetic distances.

By far, the partial test is still the most controversial application of Mantel test, and there has been a long discussion about its statistical performance in terms of Type I error and power (Raufaste and Rousset, 2001, 2002;
Castellano and Balletto, 2002; Cushman and Landguth, 2010; Harmon and Glor 2010; Legendre and Fortin, 2010; Guillot and Rousset, 2013). Actually, since its initial applications, some potential problems of low power to detect correlation and inflated Type I error in partial tests have been considered (Oden and Sokal, 1992), and different forms of permutations may provide different results depending on data characteristics (Legendre, 2000). However, some issues emerge when matrices are built upon two variables (transformed into matrices using Euclidean distances) and not multivariate distance matrices per se (such as a Nei genetic distance or pairwise $\mathrm{F}_{\mathrm{ST}}$ ). In this case there are more appropriate tools for correlating variables while taking their spatial structure into account (Dormann et al., 2007, Diniz-Filho et al., 2009; Guillot and Rousset, 2013). However, Legendre and Fortin (2010), besides indicating that other approaches have higher statistical power than the Mantel test, wrote that "....the Mantel test should not be used as a general method for the investigation of linear relationships or spatial structures in univariate or multivariate data", and "its use should be restricted to tests of hypotheses that can only be formulated in terms of distances" (see also Cushman and Landguth, 2010). Likewise, Guillot and Rousset (2013) recently found very high Type I error rates for partial Mantel tests and strongly condemned their use.

Nonetheless simulations showed that other approaches for estimating partial correlation between matrices (i.e., Redundancy Analysis based on Eigenfunction Spatial Analyses - see section below) may also have inflated Type I error rates (Legendre et al., 2005; Peres-Neto and Legendre, 2010). A simple solution to this problem with Type I error was given by Oden and Sokal (1992), who pointed out that when using partial Mantel tests it is important to be conservative and only reject the null hypothesis of no correlation if $p$ is much smaller (say, $p=0.001$ ) than the nominal level of $5 \%$. Until the development of other methods, this overall reasoning should be adopted when using partial Mantel tests.

\section{Mantel Test and Isolation-By-Distance}

Many recent studies have interpreted a significant Mantel correlation between $\mathbf{G}$ and $\mathbf{D}$ as due to Wright's Isolation-By-Distance (IBD) process. Although this is one possibility, it is hardly the only one (see Meirmans, 2012), and even a correlogram expressing a exponential-like decrease in Mantel correlations may indicate other processes creating patches of genetic variation (see Sokal and Oden, 1978a,b; Sokal and Wartenberg, 1983). Thus, it is not straightforward to link patterns to processes and, in principle, a significant Mantel test or a correlogram pattern only indicates that genetic variability is structured in geographic space. Sokal and Oden (1978b; see also Sokal and Wartenberg, 1983; and Diniz-Filho and Bini, 2012 for a historical review) proposed a more complex framework based on spatial analyses (a combination of univariate correlograms 
built with Moran's I spatial correlograms) to infer IBD, but even this framework is not unanimously accepted (see Slatkin and Arter, 1991). However, under the assumption that the processes driving genetic variation is IBD, it is possible to infer demographic and ecological parameters based on the shape of the correlograms (see Epperson, 2003; Hardy and Vekemans, 1999; Vekemans and Hardy, 2004).

Rousset (1997) showed that, under IBD, the regression of $\mathrm{F}_{\mathrm{ST}} /\left(1-\mathrm{F}_{\mathrm{ST}}\right)$ against the logarithm of geographic distances would provide a linear relationship with slope $b$ equal to

$$
\mathrm{b}=1 /\left(4 \mathrm{~N} \pi \sigma^{2}\right)
$$

and intercept $a$ equal to

$$
\mathrm{a}=-\ln (\sigma)+\gamma_{\mathrm{e}}-\ln (2)+2 \pi \mathrm{A}_{2}
$$

where $\mathrm{N}$ is the population size, $\sigma^{2}$ the variance of distance between parent and offspring ( $4 \pi \sigma^{2}$ is Wright's neighborhood area in two dimensions), $\mathrm{A}_{2}$ a constant related to the dispersal Kernel, and $\gamma_{e}$ is Euler's constant (0.5772). In practice, although it is difficult to estimate population size and dispersal distance without further experiments (capture-recapture data, for example), as it is difficult to assume $\mathrm{A}_{2}=0$ (Rousset, 1997), the theoretical derivation clearly shows how empirical relationship between matrices can provide insights on IBD parameters.

For the "Baru" dataset, the transformation of both genetic and geographic distances indicates a non-linear relationship (Figure 4), and the model with the transformations proposed by Rousset (1997) is clearly less fit. This result suggests that IBD does not apply in general, and parameter estimation associated with this process may be flawed.

\section{Alternatives to Mantel Test}

Because of the recent discussions on Mantel tests (see above), it is worthy to discuss other strategies for data analysis in the multivariate case. The overall problem in combining genetic data and geographic space, in a broad sense, is to convert the two datasets into a common "format" (i.e., vectors or distance matrices). For example, the discussions on the use of Mantel tests in the bivariate case (the correlation between two variables keeping distance constant, see Guillot and Rousset, 2013) started because space was expressed as distances, so a first idea was to transform genetic variables into distances and use a partial Mantel test (although simpler strategies to deal with spatial structures underlying two variables exist). If the data is multivariate, such as several alleles and loci used to calculate a divergence matrix, the Mantel test can be even more directly applied, because pairwise distances can be intuitively compared using this approach. However, there are other possibilities to deal with the raw data (i.e., allelic frequencies) and, because they are based on ordinations (see

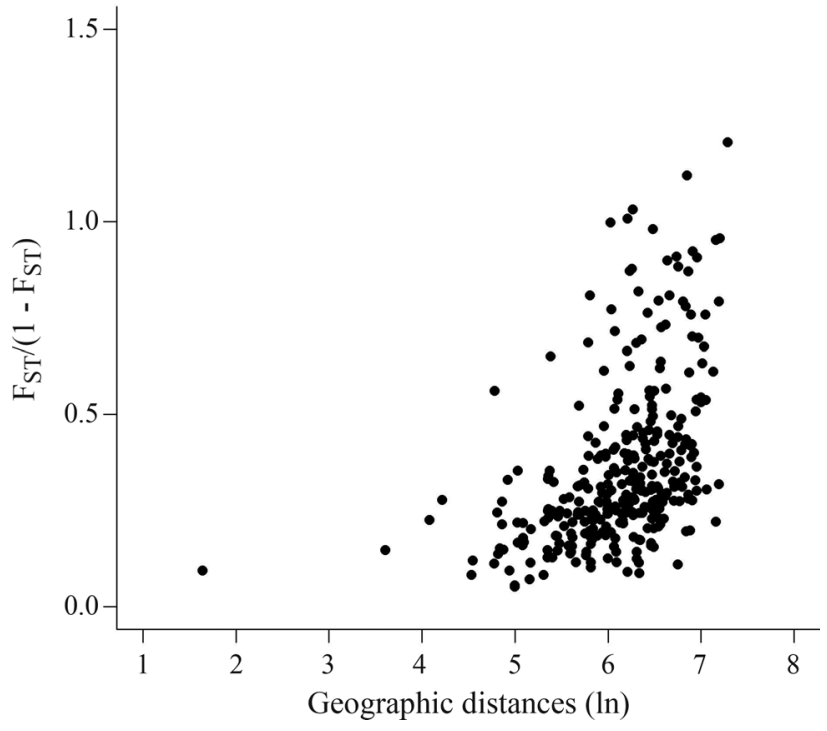

Figure 4 - Relationship between transformed $\mathrm{F}_{\mathrm{ST}}$ and logarithm of geographic distances for the 25 populations of "Baru" tree. Notice that transformation did not produce a linear relationship, supporting previous analyses showing that IBD does not apply in this case.

Legendre and Legendre, 2012), one can use scores to compare populations and not the original values per se.

The most common current alternative to the Mantel test (and partial Mantel tests) is to ordinate the genetic distances $\left(\mathrm{F}_{\mathrm{ST}}\right)$ and compare them with geographic coordinates or other vector representations of geographical distances (e.g., polynomial function of geographic coordinates). Although it is also possible to perform the analyses below based on the 52 allele frequencies directly, this would generate a Euclidean metric (Rogers) in a linear ordination, making a comparison with Mantel tests not exact (although quite close, by considering the high correlation between Nei, Rogers and $\mathrm{F}_{\text {ST }}$ pairwise distances for the "Baru"). So, we applied a Principal Coordinate Analysis (PCoA) to the $\mathrm{F}_{\mathrm{ST}}$ matrix and retained the first five axes based on a broken-stick criterion. We then used these five axes as a response matrix in a series of Redundancy Analysis (RDA) (Legendre and Legendre, 2012), and compared them with the Mantel tests already presented.

First, an RDA was carried out to analyze the spatial patterns of the genetic dataset (as summarized by the first five axes derived from $\mathrm{PCoA}$ ) using latitude and longitude as explanatory variables. This is a multivariate generalization of the linear trend surface (mTSA) analysis (see Wartenberg, 1985; Bocquet-Appel and Sokal, 1989). The coefficient of determination $R^{2}$ of the RDA was equal to 0.251 (that of the Mantel test was equal to 0.249 ). The similarity between these figures is expected by considering previous discussions about the strong linear component of genetic variation revealed by the Mantel correlograms and reflecting past range expansion.

However, the mTSA allows fitting a linear model, describing only broad-scale spatial structures. A polynomial 
function of the geographic coordinates would capture more complex patterns, but collinearity problems and low statistical power for small sample sizes make this approach less recommended. A more general approach to transform geographic space in a raw data form (i.e., variables x populations, instead of distance matrix) is to apply an eigenfunction analysis of geographic distances (or binary $\mathbf{W}$ connections) to obtain "eigenvector maps", expressing spatial relationships among populations at different spatial scales. There are several versions of this approach (see Griffith and Peres-Neto, 2006; Bini et al., 2009; Landeiro and Magnusson 2011; Diniz-Filho et al., 2009, 2012c). These methods are now collectively called Spatial Eigenfunction Analyses (SEA) and have been extensively used in ecology, and recently also gained attention from landscape geneticists (i.e., Manel et al., 2010; Manel and Holderegger, 2013).

The idea of SEA is to extract eigenvectors from geographic distances and connectivity matrices, and these eigenvectors tend to map the spatial structure among populations at different spatial scales. When allele frequencies or PCoA axes are regressed against these eigenvectors, some of them will tend to describe the spatial patterns in genetic variation. This can be done for single alleles, but here we modeled simultaneously the five axes from the PCoA of $\mathrm{F}_{\mathrm{ST}}$ matrix using an RDA, following a multidimensional approach. One of the main difficulties with this approach is to decide which spatial eigenvectors shall be used in the analyses, and several criteria can be applied. Here we followed Blanchet et al. (2008) and used a forward approach to select spatial eigenvectors. When the five axes derived from PCoA matrix were regressed against the three selected eigenvectors (1, 3 and 5), the RDA $R^{2}$ was equal to 0.362 , slightly higher than the one obtained by mTSA (because it was able to capture more complex spatial structures in genetic data beyond the overall linear trend).

Thus, the Mantel test, mTSA and SEA all showed significant correlations between $\mathbf{G}$ and $\mathbf{D}$. The magnitude of spatial pattern for $\mathbf{E}$ and $\mathbf{R}$ modeled by these different approaches was also similar (see Table 2). However, an interesting application of the ordination approach based on $\mathrm{RDA}$ is to evaluate partial relationships, providing thus an alternative to partial Mantel tests (which is important, by considering all discussions on the validity of the partial Mantel test already pointed out). Thus, a PCoA was used to map distances of matrix $\mathbf{E}$ and retaining the two axes according to the broken-stick criterion. The RDA also revealed a significant relationship between $\mathbf{G}$ and $\mathbf{E}$ (with an $R^{2}=0.215 ; \mathrm{p}<0.01$ ). By using the partial RDA it is possible to test if the genetic and environmental matrices are actually correlated after the spatial structure of both matrices is taken into account. When defining space by geographical coordinates, in the mTSA approach, the partial $R^{2}$ between $\mathbf{G}$ and $\mathbf{E}$ was equal to $0.199(\mathrm{p}<0.01)$, thus correlation between genetic and environment remained even when spatial
Table 2 - Summary of Mantel and partial Mantel tests applied to "Baru" populations, comparing effects of geographic distance (D), environmental variables $(\mathbf{E})$ and natural remnants $(\mathbf{R})$ into genetic divergence $(\mathbf{G})$ estimated by pairwise $\mathrm{F}_{\mathrm{ST}}$. Results include Mantel's correlation $r$ (and $r^{2}$, for facility of comparison with RDA results). Also provided are the $R^{2}$ of Redundancy Analysis (RDA), incorporating geographic space by spatial eigenfunction analysis (SEA) and linear multivariate trend surface (mTSA).

\begin{tabular}{lcclcc}
\hline \multirow{2}{*}{ Comparison } & \multicolumn{2}{c}{ Mantel } & & \multicolumn{2}{c}{$\mathrm{RDA}$} \\
\cline { 2 - 3 } \cline { 6 - 7 } & $\mathrm{r}$ & $\mathrm{r}^{2}$ & & $\mathrm{R}^{2}(\mathrm{SEA})$ & $\mathrm{R}^{2}(\mathrm{mTSA})$ \\
\hline GD & $0.499^{* *}$ & 0.249 & & $0.360^{* *}$ & $0.250^{* *}$ \\
ED & $0.838^{* *}$ & 0.702 & & $0.607^{* *}$ & $0.913^{* *}$ \\
RD & $0.075^{\mathrm{ns}}$ & 0.005 & & $0.337^{* *}$ & $0.349^{* *}$ \\
GE|D & $-0.248^{\mathrm{ns}}$ & 0.061 & & $0.083^{\text {ns }}$ & $0.199^{* *}$ \\
GR|D & $-0.223^{\mathrm{ns}}$ & 0.049 & & $0.018^{\mathrm{ns}}$ & $0.034^{\mathrm{ns}}$ \\
\hline
\end{tabular}

**: $\mathrm{p}<0.01 ;$ ns: non-significant at $5 \%$.

structure (i.e., the linear trend) was taken into account. However, using SEA, the $R^{2}$ between $\mathbf{G}$ and $\mathbf{E}$ (controlling for spatial interdependence) decreased to 0.083 , which was not statistically significant $(\mathrm{p}=0.23)$. Thus, when geographic space is modeled in a more appropriate way, the result from ordination was similar to that obtained by the Mantel test, which is also consistent with the fact that neutral markers, such as microsatellites, are not expected to be correlated with climatic or environmental variation.

Thus, results from RDA were similar to those provided by Mantel tests, both when comparing two matrices and when testing partial relationships (Table 2). Notice, however, that the relationship between $\mathbf{G}$ and $\mathbf{E}$ is higher for RDA than for the Mantel test (and this relationship actually disappears when $\mathbf{D}$ is taken into account). Of course, this particular example does not solve the controversies on partial Mantel tests, and other studies, using simulations, have been performed to better establish the statistical performance of these (and other) techniques. These studies concluded that, although SEA and RDA approaches may have more accurate type I and II errors, under certain conditions they can behave as badly as Mantel tests. Moreover, SEA has a more difficult component, which is the selection of eigenvectors (both in response and explanatory, in our case) to be used in the analyses. A Mantel test is simpler and can be interpreted more directly, and thus may be still valid in many cases. We believe that our empirical results reinforce that when patterns are strong and clear, techniques tend to give comparable results. In all cases, results of partial analyses should be interpreted with caution and, more likely, using the different alternatives to search for a robust and consistent outcome.

\section{Concluding Remarks}

Despite recent discussions and criticisms, we believe that the Mantel test can be a powerful approach to analyze 
multivariate data, mainly if the ecological or evolutionary hypotheses are better (or only) expressed as pairwise distances or similarities, as pointed out by Legendre and Fortin (2010). Even though, an important guideline is to always check the assumptions of linearity and homoscedasticity in the relationships between genetic divergence and other matrices (i.e., geographic distances), because such violations are actually expected under theoretical models, such as IBD. If these violations occur, a global Mantel test may be a biased description of the amount of spatial variation in the data. Mantel correlograms may be useful to overcome these problems and, at the same time, may provide a more accurate and visually appealing description of the spatial patterns in the data. Partial Mantel tests can still be applied, but using a more conservative critical level for defining their significance and, if possible, coupled with ordination and spatial eigenfunction analyses.

Finally, because of the ongoing discussions, it is important that researchers are aware of other possibilities for analyzing data, such as performed here. Although our empirical example with genetic variation in the "Baru" tree does not allow a deep evaluation of the statistical performance of these techniques and comparison with simulation-based studies, it reveals that, as is common in empirical applications, results usually converge. Thus, all these different approaches gave similar estimates of the magnitude of spatial variation in genetic variation in the "Baru" tree in the Cerrado biome, when compared with Mantel test. More importantly, the results are expected based on previous knowledge of the ecological and evolutionary processes underlying such variation.

\section{Acknowledgments}

Our research program integrating macroecology and molecular ecology of plants and the DTI fellowship to G.O. has been continuously supported by several grants and fellowships to the research network GENPAC (Geographical Genetics and Regional Planning for natural resources in Brazilian Cerrado) from CNPq/MCT/CAPES and by the "Núcleo de Excelência em Genética e Conservação de Espécies do Cerrado" - GECER (PRONEX/FAPEG/CNPq CP 07-2009). Fieldwork has been supported by Systema Naturae Consultoria Ambiental LTDA. Work by J.A.F.D.-F., L.M.B, M.P.C.T., T.N.S. and T.F.R. has been continuously supported by productivity fellowships from CNPq.

\section{References}

Balkenhol N, Waits LP and Dezzani RJ (2009) Statistical approaches in landscape genetics: An evaluation of methods for linking landscape and genetic data. Ecography 32:818830.

Bini LM, Diniz-Filho JAF, Rangel TFLVB, Akre TSB, Albaladejo RG, Albuquerque FS, Aparicio A, Araújo MB, Baselga A, Beck J, et al. (2009) Coefficients shifts in geographical ecology: An empirical evaluation of spatial and non-spatial regression. Ecography 32:193-204.

Blanchet FG, Legendre P and Borcard D (2008) Forward selection of explanatory variables. Ecology 89:2623-2632.

Bocquet-Appel JP and Sokal RR (1989) Spatial autocorrelation analysis of trend residuals in biological data. Syst Zool 38:331-341.

Borcard D and Legendre P (2012) Is the Mantel correlogram powerful enough to be useful in ecological analysis? A simulation study. Ecology 93:1473-1481.

Castellano S and Balletto E (2002) Is the partial Mantel test inadequate? Evolution 56:1871-1873.

Cushman SA and Landguth EL (2010) Spurious correlations and inference in landscape genetics. Mol Ecol 19:3592-3602.

Diniz-Filho JAF and Bini LM (2012) Thirty-five years of spatial autocorrelation analysis in population genetics: An essay in honour of Robert Sokal (1926-2012). Biol J Linn Soc 107:721-736.

Diniz-Filho JAF and Telles MPC (2002) Spatial autocorrelation analysis and the identification of operational units for conservation in continuous populations. Conserv Biol 16:924935.

Diniz-Filho JAF and Telles MPC (2006) Optimization procedures for establishing reserve networks for biodiversity conservation taking into account population genetic structure. Genet Mol Biol 29:207-214.

Diniz-Filho JAF, Nabout JC, Telles MPC, Soares TN and Rangel TFLVB (2009) A review of techniques for spatial modeling in geographical, conservation and landscape genetics. Genet Mol Biol 32:203-211.

Diniz-Filho JAF, Melo DB, Oliveira G, Collevatti RG, Soares TN, Nabout JC, Lima JS, Dobrovolski R, Chaves LJ, Naves $\mathrm{RV}$, et al. (2012a) Planning for optimal conservation of geographical genetic variability within species. Conserv Genet 13:1085-1093.

Diniz-Filho JAF, Collevatti RG, Soares TN and Telles MPC (2012b) Geographical patterns of turnover and nestednessresultant components of allelic diversity among populations. Genetica 140:189-195.

Diniz-Filho JAF, Siqueira T, Padial AA, Rangel TFLVB, Landeiro VL and Bini LM (2012c) Spatial autocorrelation allows disentangling the balance between neutral and niche processes in metacommunities. Oikos 121:201-210.

Dormann CF, McPherson J, Araújo MB, Bivand R, Bolliger J, Carl G, Davies RG, Hirzel A, Jetz W, Kissling WD, et al. (2007) Methods to account for spatial autocorrelation in the analysis of distributional species data: A review. Ecography 30:609-628.

Dutilleul P (1993) Modifying the t test for assessing the correlation between two spatial processes. Biometrics 49:305-314.

Epperson BK (2003) Geographical Genetics. Princeton University Press, Princeton, 357 pp.

Felsenstein J (2004) Inferring Phylogenies. Sinauer Press, New York, $664 \mathrm{pp}$.

Goslee SC and Urban DL (2007) The ecodist package for dissimilarity-based analysis of ecological data. J Stat Softw 22:119.

Griffith DA and Peres-Neto P (2006) Spatial modeling in ecology: The flexibility of eigenfunction spatial analyses. Ecology 87:2603-2613. 
Guillot G and Rousset F (2013) Dismantling the Mantel tests. Meth Ecol Evol 4:336-344.

Guillot G, Leblois R, Coulon A and Frantz AC (2009) Statistical methods in spatial genetics. Mol Ecol 18:4734-4756.

Hardy OJ and Vekemans X (1999) Isolation by distance in a continuous population: Reconciliation between spatial autocorrelation analysis and population genetics models. Genetics 83:145-154.

Harmon LJ and Glor RE (2010) Poor statistical performance of the Mantel test in phylogenetic comparative analyses. Evolution 64:2173-2178.

Hijmans RJ, Cameron SE, Parra JL, Jones PG and Jarvis A (2005) Very high resolution interpolated climate surfaces for global land areas. Int J Climatol 25:1965-1978.

Holsinger KE and Weir BS (2009) Genetics in geographically structured populations: Defining, estimating and interpreting $\mathrm{F}_{\mathrm{ST}}$. Nat Rev Genet 10:639-650.

Landeiro V and Magnusson W (2011) The geometry of spatial analyses: Implications for conservation biologists. Natureza \& Conservação 9:7-20.

Legendre P (2000) Comparison of permutational methods for the partial correlation and partial Mantel tests. J Statist Comput Simul 67:37-73.

Legendre P and Fortin M-J (2010) Comparison of the Mantel test and alternative approaches for detecting complex multivariate relationships in the spatial analysis of genetic data. Mol Ecol Res 10:831-844.

Legendre P and Legendre L (2012) Numerical Ecology, 3rd edition. Elsevier, Amsterdam, 990 pp.

Legendre P, Borcard D and Peres-Neto P (2005) Analyzing beta diversity: Partitioning the spatial variation of community composition data. Ecol Monogr 75:435-450.

Lessa E (1990) Multidimensional analysis of geographic genetic structure. Syst Biol 39:242-252.

Lichstein J (2007) Multiple regression on distance matrices: A multivariate spatial analysis tool. Plant Ecol 188:117-131.

Manel S and Holderegger R (2013) Ten years of landscape genetics. Trends Ecol Evol 28:614-621.

Manel S, Poncet BN, Legendre P, Gugerli F and Holderegger R (2010) Common factors drive adaptive genetic variation at different scale in Arabis alpina. Mol Ecol 19:2896-2907.

Manly BFJ (1985) The Statistics of Natural Selection. Chapman and Hall, London, 484 pp.

Manly BFJ (1997) Randomization, Bootstrap and Monte Carlo Methods in Biology. Chapman and Hall, London, 399 pp.

Mantel N (1967) The detection of disease clustering and a generalized regression approach. Cancer Res 27:209-220.

Meirmans PG (2012) The trouble with isolation-by-distance. Mol Ecol 21:2839-2846

Mielke PW (1978) Classification and appropriate inferences for Mantel and Valand's nonparametric multivariate analysis technique. Biometrics 34:277-282.

Oden N and Sokal RR (1986) Directional autocorrelation: An extension of spatial correlograms to two dimensions. Syst Zool 35:608-617.

Oden N and Sokal RR (1992). An investigation of three-matrix permutation tests. J Classif 9:275-290.

Oksanen J, Blanchet JG, Kindt R, Legendre P, Minchin PR, O'Hara RB, Simpson GL, Solymos P, Stevens MHH and
Wagner H (2012) Vegan: Community Ecology Package. R package version 2.0-5

Pellegrino KCM, Rodrigues MT, Waite AN, Morando M, Yassuda YY and Sites-Jr JW (2005) Phylogeography and species limits in the Gymnodactylus darwinii complex (Gekkonidae, Squamata): Genetic structure coincides with river systems in the Brazilian Atlantic forest. Biol J Linn Soc 85:13-26.

Peres-Neto PR and Legendre P (2010) Estimating and controlling for spatial structure in the study of ecological communities. Glob Ecol Biogeogr 19:174-184.

Perez SI, Diniz-Filho JAF, Bernal V and Gonzales PN (2010) Alternatives to the partial Mantel test in the study of environmental factors shaping human morphological variation. $\mathrm{J}$ Hum Evol 59:698-703.

R Development Core Team (2012) R: A language and environment for statistical computing, reference index version 2.15. R Foundation for statistical computing, Vienna, Austria.

Raufaste N and Rousset F (2001) Are partial Mantel tests adequate? Evolution 55:1703-1705.

Rousset F (1997) Genetic differentiation and estimation of gene flow from F-statistics under isolation-by-distance. Genetics 145:1219-1228.

Slatkin M and Arter HE (1991) Spatial autocorrelation methods in population genetics. Am Nat 138:499-517.

Smouse PE, Long JC and Sokal RR (1986). Multiple regression and correlation extensions of the Mantel test of matrix correspondence. Syst Zool 35:627-632.

Soares TN, Melo DB, Resende LV, Vianello RP, Chaves LJ, Collevatti RG and Telles MPC (2012) Development of microsatellite markers for the Neotropical tree species Dipteryx alata (Fabacea). Amer J Bot 99:72-73.

Sokal RR (1979) Testing statistical significance of geographic variation patterns. Syst Zool 28:227-232.

Sokal RR and Oden NL (1978a) Spatial autocorrelation in biology. 1. Methodology. Biol J Linn Soc 10:199-228.

Sokal RR and Oden NL (1978b) Spatial autocorrelation in biology. 2. Some biological implications and four applications of evolutionary and ecological interest. Biol J Linn Soc 10:229-249

Sokal RR and Wartenberg DE (1983) A test of spatial autocorrelation analysis using an isolation-by-distance model. Genetics 105:219-237.

Sokal RR, Smouse P and Neel JV (1986) The genetic structure of a tribal population, the Yanomama indians. XV. Patterns inferred by autocorrelation analysis. Genetics 114:259-287.

Sokal RR, Oden NL, Legendre P, Fortin M-J, Kim J and Vaudor A (1989) Genetic differences among language families in Europe. Am J Phys Anthropol 79:489-502.

Sokal RR, Oden NL, Walker J and Waddle DM (1997) Using distance matrices to choose between competing theories and an application to the origin of modern humans. J Hum Evol 32:501-522.

Vekemans X and Hardy OJ (2004) New insights from fine-scale spatial genetic structure analyses in plant populations. Mol Ecol 13:921-935.

Wagner HH and Fortin MJ (2013) A conceptual framework for the spatial analysis of landscape genetic data. Conserv Genet $14: 253-261$ 
Wartenberg D (1985) Canonical trend surface analysis: A method for describing geographic patterns. Syst Zool $34: 259-279$.

\section{Internet Resources}

WorldClim, http://www.worldclim.org (September 13, 2013).
Vegan: Community Ecology Package. R package version 2.0-5. http://CRAN.R-project.org/package=vegan. (June 20, 2012).

Associate Editor: Alexandre Rodrigues Caetano

License information: This is an open-access article distributed under the terms of the Creative Commons Attribution License, which permits unrestricted use, distribution, and reproduction in any medium, provided the original work is properly cited. 